\title{
Structural Insights into Poly(Heptazine Imides): A Light-Storing Carbon Nitride Material for Dark Photocatalysis
}

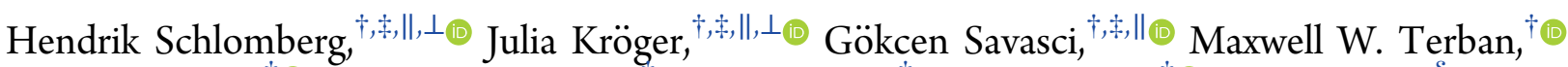 \\ Sebastian Bette, ${ }^{\dagger}$ I Igor Moudrakovski, ${ }^{\dagger}$ Viola Duppel, ${ }^{\dagger}$ Filip Podjaski, ${ }^{\dagger}$ Renée Siegel, ${ }^{\S}$ \\ Jürgen Senker, ${ }^{\S}$ Robert E. Dinnebier, ${ }^{\dagger}$ Christian Ochsenfeld, ${ }^{\ddagger}, \|_{\odot}$ and Bettina V. Lotsch ${ }^{*}, \dagger,+, \|_{0}$ \\ ${ }^{\dagger}$ Max-Planck-Institut für Festkörperforschung, Heisenbergstraße 1, 70569 Stuttgart, Germany \\ ${ }^{\ddagger}$ Department Chemie, Ludwig-Maximilians-Universität München, Butenandtstraße 5-13, 81377 München, Germany \\ ${ }^{\S}$ Inorganic Chemistry III and Northern Bavarian NMR Centre, University of Bayreuth, Universitätsstr. 30, 95440 Bayreuth, \\ Germany \\ "Center for Nanoscience and Cluster of excellence e-conversion, Schellingstraße 4, 80799 München, Germany
}

Supporting Information

\begin{abstract}
Solving the structure of carbon nitrides has been a longstanding challenge due to the low crystallinity and complex structures observed within this class of earth-abundant photocatalysts. Herein, we report on two-dimensional layered potassium poly(heptazine imide) (K$\mathrm{PHI}$ ) and its proton-exchanged counterpart (H-PHI), obtained by ionothermal synthesis using a molecular precursor route. We present a comprehensive analysis of the in-plane and three-dimensional structure of PHI. Transmission electron microscopy and solid-state NMR spectroscopy, supported by quantum-chemical calculations, suggest a planar, imidebridged heptazine backbone with trigonal symmetry in both $\mathrm{K}-\mathrm{PHI}$ and $\mathrm{H}$ PHI, whereas pair distribution function analyses and X-ray powder diffraction using recursive-like simulations of planar defects point to a structure-directing function of the pore content. While the out-of-plane structure of K-PHI exhibits a unidirectional layer offset, mediated by hydrated potassium ions, H-PHI is characterized by a high degree of stacking faults due to the weaker structure directing influence of pore water. Structureproperty relationships in PHI reveal that a loss of in-plane coherence, materializing in smaller lateral platelet dimensions and increased terminal cyanamide groups, correlates with improved photocatalytic performance. Size-optimized H-PHI is highly active toward photocatalytic hydrogen evolution, with a rate of $3363 \mu \mathrm{mol} / \mathrm{gh} \mathrm{H}_{2}$ placing it on par with the most active carbon nitrides. K- and H-PHI adopt a uniquely long-lived photoreduced polaronic state in which light-induced electrons are stored for more than $6 \mathrm{~h}$ in the dark and released upon addition of a Pt cocatalyst. This work highlights the importance of structureproperty relationships in carbon nitrides for the rational design of highly active hydrogen evolution photocatalysts.
\end{abstract}

\section{INTRODUCTION}

A new class of (semi)crystalline two-dimensional (2D) carbon nitrides has emerged over the past few years, which exhibits outstanding photocatalytic performance ${ }^{1-3}$ along with intriguing photophysical properties, such as the potential for timedelayed fuel production ("dark photocatalysis"), ${ }^{4}$ which also make them attractive candidate materials for solar batteries. ${ }^{5} \mathrm{~A}$ hallmark of this carbon nitride family is its ability to store electrons in the form of a long-lived photoreduced state, ${ }^{4,6,7}$ which allows for the separation of light absorption (the light reaction) and catalytic conversion (the dark reaction) akin to natural photosynthesis. This material has been postulated to exhibit a 2D poly(heptazine imide) (PHI)-based structure, but so far a comprehensive elucidation of its local and long-range structure is lacking. PHI has been synthesized by both, molecular precursor approaches ${ }^{8,9}$ as well as top down strategies starting from the well-known melon polymer. ${ }^{2,10}$ And yet, even though the synthesis procedures may vary widely, the use of salt melts, i.e., ionothermal synthesis, seems to be vital to all of these approaches. ${ }^{2,8-10}$

Ionothermal synthesis routes often yield fundamentally different products as compared to high-temperature solidstate approaches. A prominent example is the synthesis of melon, which is often referred to as graphitic carbon nitride, g$\mathrm{C}_{3} \mathrm{~N}_{4}$. Although the solid-state synthesis of melon yields a onedimensional heptazine-based polymer, 2D networks are typically formed by a salt-melt approach using the same types of precursors. Under these conditions, the ionic flux and its components, typically alkali metal halides, serve as a hightemperature solvent and structure-directing agent at the same time. ${ }^{11-13}$ The structure-directing influence of alkali ions has been observed by Savateev et al. and is also known to be the

Received: June 5, 2019

Revised: August 9, 2019

Published: August 12, 2019 


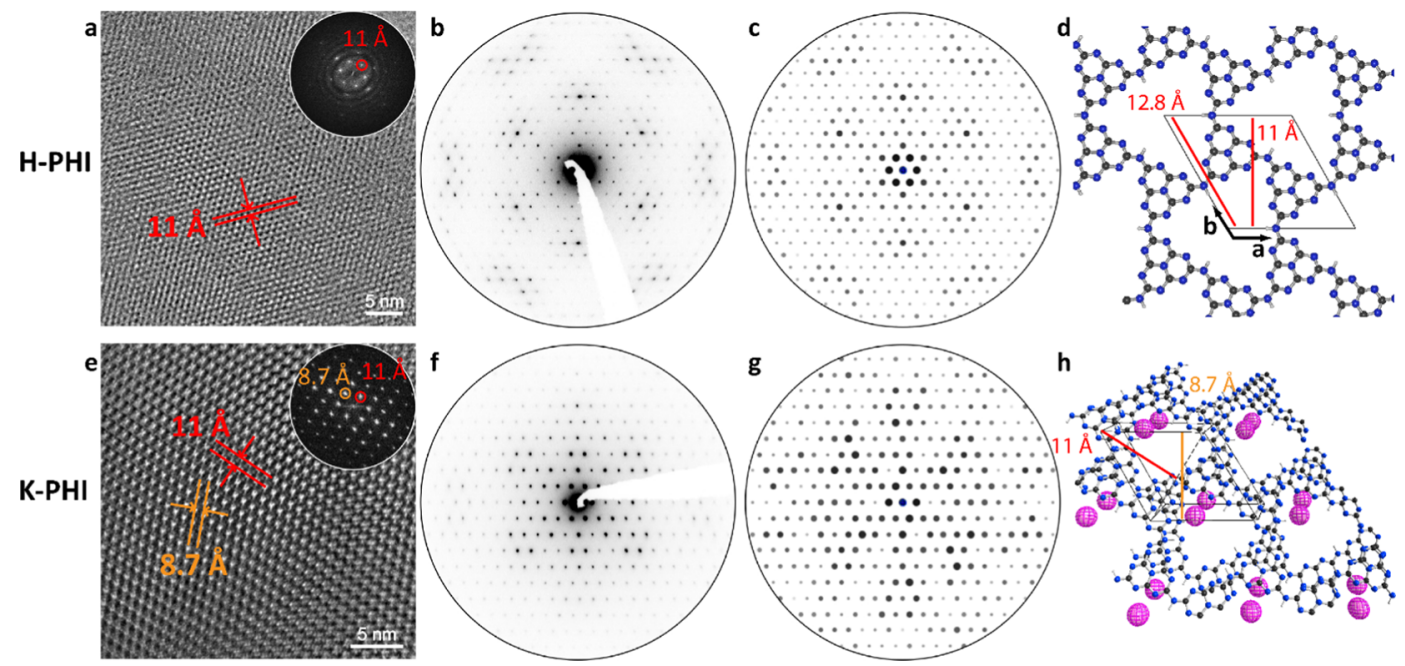

Figure 1. (a) High-resolution TEM image of H-PHI clearly revealing lattice fringes over extended areas; (b) SAED pattern and (c) simulated SAED pattern of the [001] zone axis of H-PHI, based on a theoretical trigonal cell model depicted in $(\mathrm{d})$. (e-g) cover the corresponding figures for K-PHI. Insets show the FFT confirming the 6-fold symmetry of the diffraction pattern for H-PHI and the reduced monoclinic layer symmetry for K-PHI, respectively. (h) Structural model obtained from Rietveld refinement for K-PHI (see Figure 3b); water molecules inside the pores were omitted for better visibility.

governing factor during the formation of secondary building units in zeolites. ${ }^{9,14}$ Interestingly, the composition of the melt also seems to have a profound influence on the type of carbon nitride backbone-triazine or heptazine-based-that is formed during the reaction. ${ }^{8}$

Herein, we have used a molecular building block approach to synthesize highly crystalline potassium containing PHI terminated by cyanamide $\left(\mathrm{NCN}^{-}\right)$-functional groups, abbreviated as potassium PHI (K-PHI), and its metal-free counterpart H-PHI. A comprehensive investigation of their local and long-range structures has been carried out through a combination of electron diffraction (ED) and transmission electron microscopy (TEM) imaging, solid-state nuclear magnetic resonance (ssNMR) spectroscopy, supported by quantum-chemical calculations, X-ray powder diffraction (XRPD), and pair distribution function analysis (PDF). We show that both materials feature an extended 2D network constituted by tri-s-triazine (heptazine) units connected via imide bridges. These layers assemble in either ordered or disordered long-range stacking relationships driven by interactions with the pore content, resulting in hitherto unknown structural modifications in this PHI family.

\section{RESULTS AND DISCUSSION}

The ionothermal bottom-up synthesis of PHI starts from the molecular precursors potassium melonate, dicyandiamide, and potassium thiocyanate (KSCN), which are heated under their autogenous pressure in an ampoule to yield highly crystalline potassium poly(heptazine imide) (K-PHI), terminated with $\mathrm{NCN}$-functional groups. In contrast to other PHI-derived materials (mainly synthesized from $\mathrm{LiCl} / \mathrm{KCl}$ eutectics), the herein discussed synthesis is based on $\mathrm{KSCN}$ as the salt component and hence flux. When K-PHI is treated with dilute acid, it is converted into the proton-bearing $\mathrm{H}$-PHI, thus pointing to the Brønsted acid-base character of the polymer. ${ }^{9}$ Note that the reaction is insensitive to which acid (e.g., $\mathrm{HCl}$, $\mathrm{H}_{3} \mathrm{PO}_{4}, \mathrm{H}_{2} \mathrm{SO}_{4}$, or $\mathrm{HClO}_{4}$ ) is used for the transformation (Figures S50-S52). In the following text, we elucidate the local structure of K-PHI and H-PHI by solid-state NMR spectroscopy and characterize the $2 \mathrm{D}$ projection of the structure by means of TEM, whereas XRPD, PDF analyses, and recursive-like simulations of planar defects lead to the classification of the material in the third dimension.

TEM Analysis. The TEM image in Figure 1a reveals the sheetlike morphology of the crystallites and high crystallinity of the carbon nitride backbone over a large area of up to several $100 \mathrm{~nm}$ (for overview TEM images see Supporting Information Figures S1 and S3). The lattice fringes correspond to an interval of $11 \AA$ within the layer planes, illustrated by the high-resolution (HR) TEM image in Figure 1a. The good agreement between the measured (Figure $1 \mathrm{~b}$ ) and simulated (1c) selected area electron diffraction (SAED) patterns along the [001] zone axis based on the structural model shown in Figure 1d indicates trigonal layer symmetry of the PHI backbone in H-PHI. The fast Fourier transformation (FFT) obtained from large crystallites (Figure 1a, inset) also fits the depicted SAED pattern. The 100 lattice spacing of $11 \AA$ corresponds to the pore-to-pore distance of $12.8 \AA$ and is consistent with a hexagonal arrangement of trigonal pores and a $2 \mathrm{D}$ network topology, similar to that found in the triazine analogue poly(triazine imide) (PTI). ${ }^{11,12}$ The TEM data suggest that ideal H-PHI layers crystallize with trigonal symmetry, forming a "graphitic" 2D framework of imidebridged heptazine building units akin to melamine-intercalated PHI, which crystallizes in the space group $P 31 \mathrm{~m} .{ }^{15}$ Particularly in the H-PHI case, slight intensity deviations from the perfect hexagonal distribution of the electron diffraction spots (Figure 1b) and the fact that the diffraction data of $\mathrm{H}-\mathrm{PHI}$ are relatively tolerant against tilting of the specimen for up to $\pm 10^{\circ}$ indicate structural disorder along the [001] zone axis. Intensity deviations might also arise from losing pore content (i.e., water) in ultra high vacuum and local heating through the electron beam, which may induce on-spot incipient degradation and enable the layers to shift to the eclipsed case, as also seen in thermogravimetric XRPD experiments in Figure S27. The diffraction spots of K-PHI (Figure 1f, [001] zone axis) imply that the material exhibits monoclinic layer symmetry. The SAED pattern can be reproduced by ED simulation with 

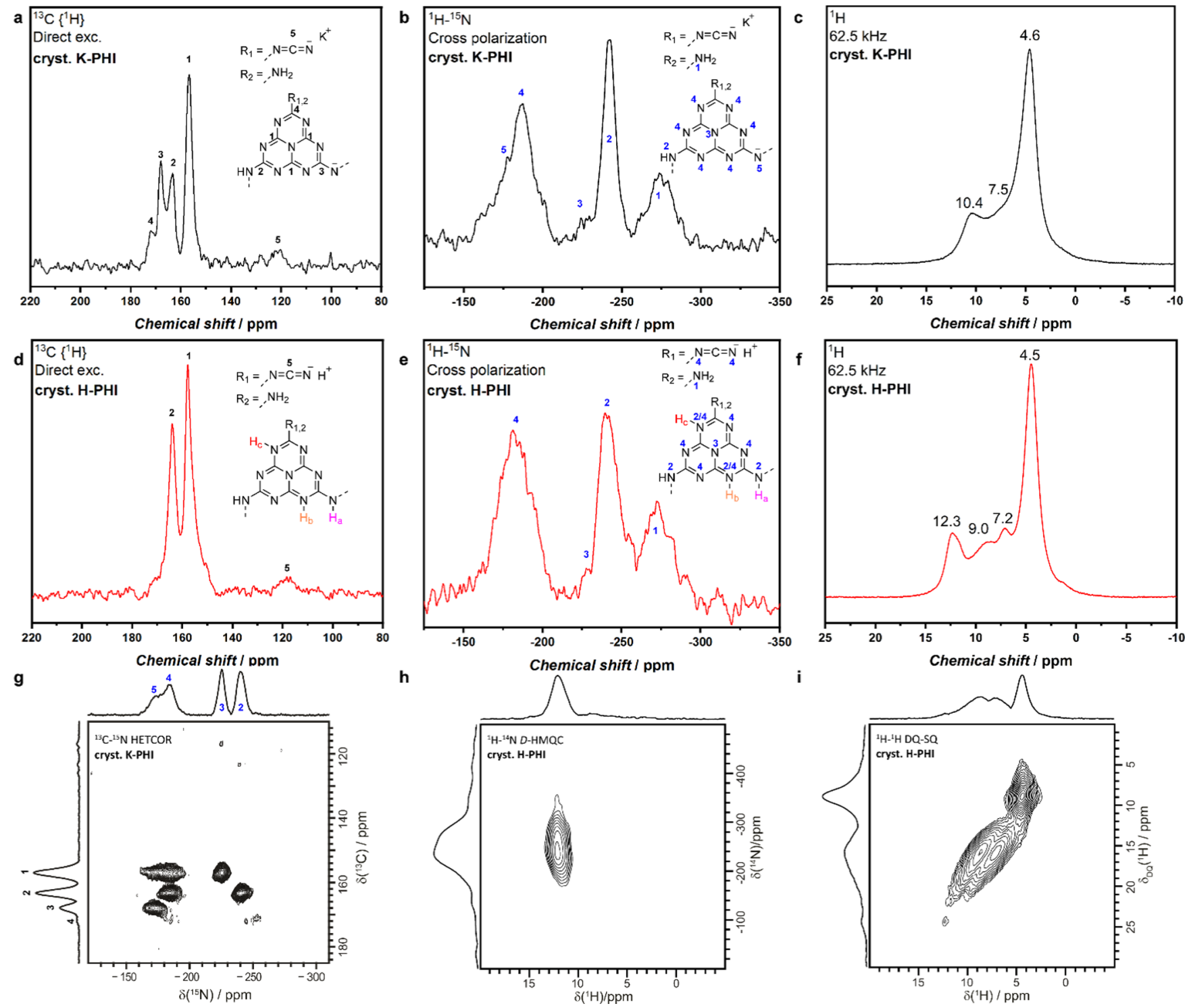

Figure 2. (a, d) ${ }^{13} \mathrm{C}$ direct and (b, e) ${ }^{15} \mathrm{~N}$ cross-polarization and (c, f) ultrafast ${ }^{1} \mathrm{H}$ magic angle spinning (MAS) solid-state NMR spectra of K-PHI and $\mathrm{H}$-PHI. The contact times of $\mathrm{CP}{ }^{15} \mathrm{~N}$ NMR spectra were both set to $4 \mathrm{~ms}$. Insets show the proposed structures and their NMR assignments in black for carbon atoms and in blue for nitrogen atoms. In the case of $\mathrm{H}-\mathrm{PHI}$, the different possible protonation sites are labeled $\mathrm{H}_{a}, \mathrm{H}_{\mathrm{b}}, \mathrm{H}_{c}$; however, coexistence of several different tautomers is possible with the majority of $\mathrm{NH}$ groups still located at bridges. $(\mathrm{g}){ }^{13} \mathrm{C}-{ }^{15} \mathrm{~N}$ dynamic nuclear polarization (DNP) HETCOR NMR spectrum of K-PHI, ${ }^{1} \mathrm{H}-{ }^{14} \mathrm{~N}$ dipolar heteronuclear multiple-quantum coherence, and ${ }^{1} \mathrm{H}-{ }^{1} \mathrm{H}$ DQ-SQ spectra of H-PHI (h, i).

corresponding distances of $11 \AA$ for the $\overline{1} 10$ and $8.7 \AA$ for the 100 reflection (Figure $1 \mathrm{~g}$ ). The HR-TEM image (Figure 1e) also points toward the existence of large crystalline domains, with the FFT (inset) reproducing the pattern obtained from the simulation in Figure 1g. The difference in symmetry suggests an altered stacking behavior of the PHI backbones in K-PHI and H-PHI, likely driven by the pore constituents (water in $\mathrm{H}-\mathrm{PHI}$ or potassium ions in K-PHI). Note that the water content nearly doubles from 10 wt \% for K-PHI to 20 wt $\%$ for H-PHI, determined by combustion analysis and inductively coupled plasma (Tables S8 and S9).

Solid-State NMR Spectroscopy. To probe the local structure of $\mathrm{PHI}$, especially the intralayer connectivity and the distribution of cations within the pores, ${ }^{13} \mathrm{C},{ }^{15} \mathrm{~N},{ }^{14} \mathrm{~N}$, and ${ }^{1} \mathrm{H}$ ssNMR spectra were recorded for K-PHI and compared with those of $\mathrm{H}$-PHI. Figure $2 \mathrm{a}-\mathrm{i}$ shows the ${ }^{13} \mathrm{C}$ direct excitation, ${ }^{15} \mathrm{~N}$ cross-polarization (CP) and ${ }^{1} \mathrm{H}$ ssNMR spectra of K-PHI (top row) and H-PHI (middle row), as well as the ${ }^{13} \mathrm{C}-{ }^{15} \mathrm{~N}$ $2 \mathrm{D}$ heteronuclear correlation (HETCOR) ${ }^{16}$ spectrum of $\mathrm{K}$ PHI, the ${ }^{1} \mathrm{H}-{ }^{14} \mathrm{~N}$ 2D HETCOR, and 2D ${ }^{1} \mathrm{H}$ doublequantum-single-quantum (DQ-SQ $)^{17}$ spectra of $\mathrm{H}-\mathrm{PHI}$ (bottom row). Various model systems of single PHI pores with different substitution patterns and positions were designed and optimized on the PBE0-D3/def2-TZVP ${ }^{18-21}$ level of theory to then calculate NMR chemical shifts on the B97-2/pcsSeg- $2^{22,23}$ level of theory on optimized pore geometries (Figures S18-S24) to develop a better understanding of the experimental data and to support the assignment.

The direct excitation ${ }^{13} \mathrm{C}$ NMR experiment shows five distinct signals for K-PHI, which can be assigned according to the inset in Figure 2a. Like in melem, ${ }^{24}$ the central carbon atom $(\mathrm{C} 1)$ in the heptazine unit exhibits a chemical shift of $157 \mathrm{ppm}$. The signal at $164 \mathrm{ppm}$ is attributed to a peripheral 
carbon atom next to a $\mathrm{NH}$ group $(\mathrm{C} 2)$ as its signal is the most intense in the ${ }^{13} \mathrm{C}$ cross-polarization experiment confirming its proximity to a proton (Figure S12). Additionally, the ${ }^{13} \mathrm{C}-{ }^{15} \mathrm{~N}$ 2D HETCOR spectrum shows distinctly that $\mathrm{C} 2$ correlates with the $\mathrm{NH}$ group at $-242 \mathrm{ppm}$ (Figure $2 \mathrm{~g}$ ), identifying this $\mathrm{NH}$ as the connecting bridge between two heptazine units. The ${ }^{13} \mathrm{C}$ NMR signal at $168 \mathrm{ppm}$ arises from a carbon atom next to a deprotonated imide bridge (C3). Indeed, this C3 is only connected to nitrogen atoms with a chemical shift at $-173 \mathrm{ppm}$ (Figure 2g), a typical value for nonprotonated nitrogen. In the case of an attached NCN moiety, the neighboring heptazine carbon atom (C4) is shifted downfield to $172 \mathrm{ppm}$ whereas the carbon atom of the terminal NCN group (C5) is found at $120 \mathrm{ppm}$, which is likewise observed for cyanamide in molecular potassium melonate. ${ }^{25}$ However, according to the integrated intensities in NMR spectra, these cyanamide groups are only present in about every second to fifth (batch variations) heptazine ring in K-PHI. Assuming only terminating cyanamide groups at the edges of the crystallites, especially for large crystals in the range of $100 \mathrm{~nm}$, the expected NCN content would be lower than the observed amount. However, additional cyanamide could have been introduced as point defects within the PHI sheet. Such a point defect is equivalent to a missing heptazine unit, leveraging additional internal NCN termini, since the former $\mathrm{NH}$ bridges become terminating $\mathrm{NCN}$ groups inside the PHI layer. As discussed in more detail in the Supporting Information (SI) (Figure S9), the concentration of these defects has been estimated to be approx. 5-12\%. The number of NCN groups seems to be reduced upon the transformation to H-PHI, which can be attributed to partial hydrolysis of the functional group, resulting in an increased number of terminal $\mathrm{NH}_{2}$ groups or urea terminations.

${ }^{15} \mathrm{~N}$ CP MAS NMR of K-PHI confirms the presence of $\mathrm{NH}$ groups at $-242 \mathrm{ppm}(\mathrm{N} 2$, inset Figure $2 \mathrm{~b}$ ) bridging the heptazine units, as well as a small amount of terminal $\mathrm{NH}_{2}$ groups at $-273 \mathrm{ppm}(\mathrm{N} 1)$ (note that the intensity of the $\mathrm{NH}_{2}$ CP signal is grossly overestimated). The ${ }^{15} \mathrm{~N}$ direct excitation spectra (Figure S11) show negligible (below detection limit) amounts of these $\mathrm{NH}_{2}$ groups. The central nitrogen atom at $-229 \mathrm{ppm}$ (N3) has a very low intensity in the CP experiments, as the polarization transfer is very poor. This assignment is confirmed by the ${ }^{13} \mathrm{C}-{ }^{15} \mathrm{~N} 2 \mathrm{D}$ correlation experiment (Figure $2 \mathrm{~g}$ ) in which the central nitrogen is only connected to the carbon at $157 \mathrm{ppm}$ (C1). The peripheral nitrogen atoms at -160 to $-200 \mathrm{ppm}$ (N4) lie in the typical range of shifts known from heptazine-derived molecules. ${ }^{24}$ Interestingly, the best agreement with our quantum-chemical models is found by assuming the "naked" anionic N-bridge to be screened by a potassium ion (Figures S23). In this case, calculations suggest that the signal of the anionic bridge (N5) lies at $-173 \mathrm{ppm}$ and is thus beneath the shifts of the heptazine core. Placing the potassium ion in the center of the pore (Figure S24), a shift at $>-140 \mathrm{ppm}$ is calculated for the bridging nitrogen atom, which is not found in our measurements. Thus, our data suggest that $\mathrm{K}^{+}$, presumably with a partial hydration shell, is located off-center and moved toward the polymeric backbone.

Although the structure of the acid-treated polymer H-PHI is largely similar, some distinct changes in the NMR spectra accompany the transformation. The NMR data of H-PHI support the assumption that protonation occurs predominantly at formerly negatively charged bridging nitrogen atoms $\left(\mathrm{H}_{a}\right.$ inset Figure 2d). Due to the conjugated charge in the network, however, protonation may also occur directly at the heptazine ring. Quantum-chemical calculations have identified $\mathrm{H}_{\mathrm{b}}$ and $\mathrm{H}_{\mathrm{c}}$ (inset Figure $2 \mathrm{~d}$ ) as potential protonation sites. The corresponding calculated chemical shift of these ring protonations are -247 and $-261 \mathrm{ppm}$, respectively, which is in agreement with the upfield shift of the NH-group signal in Figure 2e. Molecular heptazine-based compounds such as cyameluric acid or protonated calcium melonate support this possibility, as they also bear the proton at the heptazine ring. ${ }^{26,27}$ However, the overall low absolute amount of $\mathrm{NH}$ sites at the ring, along with the coexistence of different protonation sites and expected similar chemical shifts (see calculated pore models in Figures S18, S19, and S22) for this group compared with the bridging $\mathrm{NH}$, does not allow for an unambiguous resolution of the protonation sites.

In direct excitation ${ }^{13} \mathrm{C}$ NMR of protonated samples $(\mathrm{H}$ $\mathrm{PHI}$ ), the previously visible signal C3 next to a deprotonated bridge now disappears, hinting that this nitrogen has been protonated, which would result in a chemical shift similar to that of $\mathrm{C} 2$. Indeed, the relative intensity of $\mathrm{C} 2$ increases for $\mathrm{H}$ $\mathrm{PHI}$ in the directly detected ${ }^{13} \mathrm{C}$ spectrum. The resonance of C5 at 120 ppm slightly shifts upfield to $117 \mathrm{ppm}$ as compared to that of K-PHI, which is also mapped by our calculations (Figure S18). The carbon atom next to the protonated NCN group is now located beneath the signal of $\mathrm{C} 2$. The ${ }^{15} \mathrm{~N} \mathrm{CP}$ MAS experiment yields a picture for $\mathrm{H}$-PHI very similar to that for K-PHI. However, the $\mathrm{NH}$ group at $-242 \mathrm{ppm}$ is broadened toward higher fields in H-PHI and the central nitrogen in the heptazine unit is slightly upfield-shifted to $-233 \mathrm{ppm}$ as well. Additionally, a shoulder of the carbon signal at $157 \mathrm{ppm}$ toward higher fields is also observed. These small changes could be related to interactions with protons of water in close proximity to nitrogen atoms on the $s$-heptazine ring and the possibility of $\mathrm{NH}$ located at the ring.

The ${ }^{1} \mathrm{H}$ NMR measurements of K-PHI and H-PHI (Figure $2 c, f)$ help to give a clearer picture on the role of water and protons within those networks. Both spectra exhibit an intense signal at about $4.5 \mathrm{ppm}$, which is assigned to relatively free water inside the pores. Several additional overlapping peaks can be observed between 5 and 12.3 ppm for H-PHI. From the ${ }^{1} \mathrm{H}-{ }^{14} \mathrm{~N} 2 \mathrm{D}$ correlation spectra, the proton of the bridging $\mathrm{NH}$ can be assigned to a chemical shift of about 10.4 ppm for KPHI (Figure S14b) and $12.3 \mathrm{ppm}$ for H-PHI (Figure 2h), confirming that the majority of the bridging nitrogen atoms are protonated. Note that this low-field shifted resonance varies with the amount of water present in the sample. For a "dry" $\mathrm{H}$ PHI sample (vacuum-dried at $120{ }^{\circ} \mathrm{C}$ for 2 days), this peak shifts upfield to about $7 \mathrm{ppm}$, as shown by its ${ }^{1} \mathrm{H}-{ }^{14} \mathrm{~N} 2 \mathrm{D}$ HETCOR spectrum (Figure S14a). This is in agreement with the calculations, hinting at water as a factor that significantly influences the proton NMR shifts. A calculated model pore with one water molecule interacting with an imide bridge leads to a shift of the $\mathrm{NH}$ proton from $8 \mathrm{ppm}$ (without water) to roughly $11 \mathrm{ppm}$ (with water), which is close to the experimental results (cf. Figures S19 and S20). In H-PHI, this proton signal is downfield-shifted to $12.3 \mathrm{ppm}$ compared to that of the potassium-containing anionic network (10.4 ppm), thus indicating more acidic protons for H-PHI. The ${ }^{1} \mathrm{H}$ NMR resonances between the free water and the $\mathrm{NH}$ bridging peaks (between 5 and $12 \mathrm{ppm}$ for $\mathrm{H}-\mathrm{PHI}$ ) reveal no strong bonding to the network and consequently show no correlation with any nitrogen in the ${ }^{1} \mathrm{H}-{ }^{14} \mathrm{~N} 2 \mathrm{D}$ HETCOR 

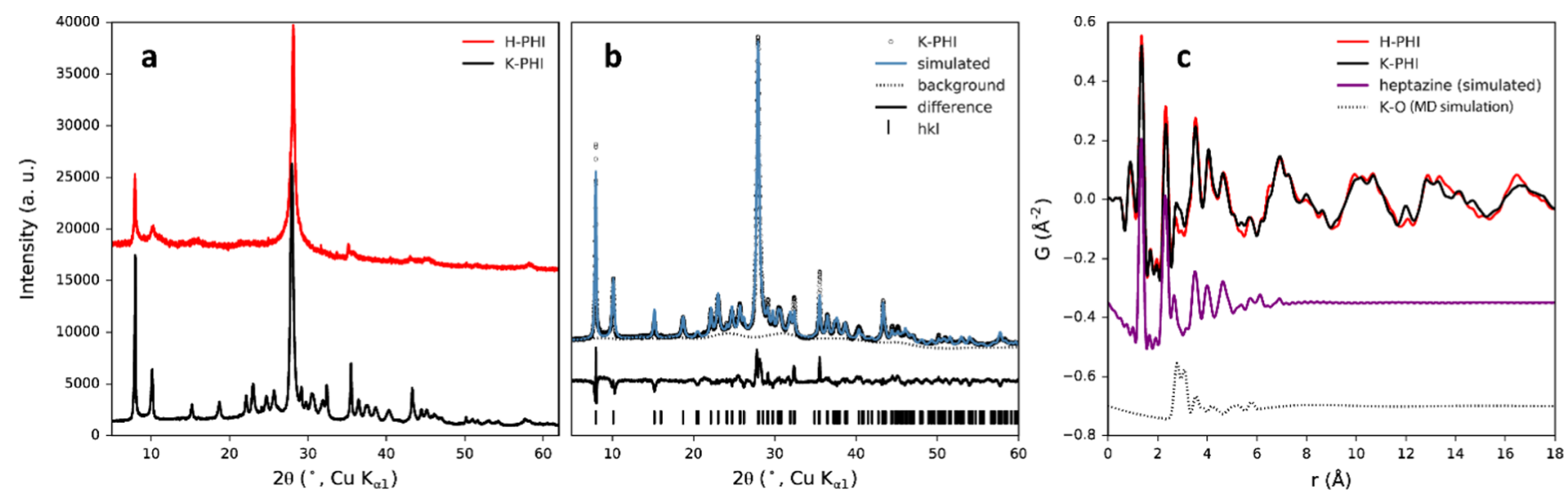

Figure 3. (a) XRPD patterns of H-PHI and K-PHI measured using $\mathrm{Cu} \mathrm{K} \alpha_{1}$ radiation, (b) Rietveld refinement for K-PHI, and (c) experimental PDFs for K-PHI and H-PHI, and simulated ones from a single heptazine molecule and from a model of potassium-oxygen distances derived from molecular dynamics (MD) simulations.

spectrum and hence are assigned to additional water inside the pore. The ${ }^{1} \mathrm{H}-{ }^{1} \mathrm{H}$ 2D DQ-SQ spectrum of $\mathrm{H}$-PHI (Figure 2i) provides both a higher resolution for ${ }^{1} \mathrm{H}$ and information on proximity between these protons. On the diagonal we observe the self-correlation peak of free water at $4.5 \mathrm{ppm}$ and also the bridging $\mathrm{NH}$ self-correlation, although this second one is less intense due to a large distance between the $\mathrm{NH}$ units. In addition, we observe a quite intense correlation between one ${ }^{1} \mathrm{H}$ at 7.2 and one at $9.0 \mathrm{ppm}$. From the DQ-SQ experiment acquired with different recoupling times (Figure S15), these two protons are close to each other (their distance is similar to the ${ }^{1} \mathrm{H}-{ }^{1} \mathrm{H}$ distance in water). This would hint at a water molecule with each of its two protons creating hydrogen bonds to nitrogen atoms on the heptazine ring. Similar protons are also observed in the case of K-PHI (Figure S16), i.e., also in close contact to the network. In general, ssNMR spectroscopy, in combination with our calculated pore models, corroborates the existence of a heptazine-based interconnected 2D network for K-PHI and H-PHI partially functionalized by NCN groups. In both materials, the network strongly interacts with water and hydrated potassium, respectively. This interplay represents a key factor for explaining the three-dimensional (3D) structure, as presented in the following sections.

X-Ray Powder Diffraction and PDF Analysis. XRPD patterns show significant peak broadening for H-PHI, whereas K-PHI exhibits higher crystallinity and was therefore used as the starting point for modeling (Figure 3a). In line with the TEM data, the Bragg peaks could be indexed with a triclinic unit cell, which was confirmed by Pawley refinement ${ }^{28}$ to be pseudohexagonal with $a=b=12.78 \AA, c=4.31 \AA, \alpha=\beta=$ $109.63^{\circ}$ and $\gamma=120^{\circ}, R_{\mathrm{wp}}=3.87 \%$. The structure model for the Rietveld refinement ${ }^{29}$ (Figure $3 \mathrm{~b}$ ) was based upon the previously solved structure of PTI, reimagined for PHI sheets. ${ }^{11,12}$ The cell metric indicates a single layer per cell, which is stacked in a slip-stacked fashion, given the non- $90^{\circ} \alpha$ / $\beta$ angles. A planar heptazine dimer was defined as a rigid body and oriented in the $a b$-plane, forming large triangular pores with two heptazine units on each side and an interlayer distance of approximately $3.2 \AA$, in agreement with the prominent 001 stacking reflection at ca. $28^{\circ} 2 \theta$. Reasonable relative peak intensities could only be obtained by including $\mathrm{K}$ and $\mathrm{O}$ atoms (i.e., water molecules) representing the pore content, indicating that the pores are indeed filled. Further details on Pawley and Rietveld analyses can be found in Figures S28-S31.

$\mathrm{H}-\mathrm{PHI}$ appears to be structurally related through welldefined $1 \overline{1} 0,010 / 100,001$, and $32 \overline{1} / 23 \overline{1}$ reflections and could be indexed by Pawley refinement with a similar cell (Figure S29). However, a suitable Rietveld refinement for H-PHI was not possible due to significant peak broadening. Since NMR and TEM data suggest an intact layer constitution of H-PHI, this broadening is attributed to planar defects occurring as stacking fault disorder that developed during $\mathrm{K}^{+}$removal, which is discussed in the next section.

To gain further insights into the local structure, PDFs (Figures S32 and S33) were obtained from synchrotron X-ray total scattering data for H-PHI and K-PHI, in Figure 3c. A direct comparison shows highly similar local structures in both compounds. The heptazine units are present and conformationally rigid. Broad, interlayer peaks repeating with a wavelength of $\sim 3.19 \AA$ for K-PHI and $\sim 3.17 \AA$ for H-PHI indicate that the sheets are stacked with high fidelity, on the order of at least several hundred Angstroms, shown in Figures S32 and S33. The lack of longer wavelength modulations expected from periodic porous channels indicates that the pores are populated by uncorrelated electron density. Small differences in medium-range, low-amplitude features indicate possible differences in local ordering of pore content and neighboring layers, whereas more drastic long-range deviation in the behavior of the interlayer peaks provides additional evidence of differences in the stacking behavior. Model refinements to the K-PHI and H-PHI PDFs (Figures S34S36) were consistent with the results from Rietveld refinements, as shown in Figures S30 and S31. In terms of shortrange ordering, the most notable difference between $\mathrm{H}$-PHI and $\mathrm{K}-\mathrm{PHI}$ is the increase in peak intensity around $2.7-3.3 \AA$, which correlates with an increase in $\mathrm{K}^{+}$(Figure S37) concentration. This feature is connected to potassium-oxygen distances found in water-solvated potassium ions observed via MD simulations (Figure S25), providing direct evidence of water $-\mathrm{K}^{+}$coordination within the pores. Notably, in all Rietveld and PDF refinements, $\mathrm{K}^{+}$ions consistently moved off center, in-plane, toward one corner of the triangular pore. Complexation of $\mathrm{K}^{+}$ions with azines has previously been shown favorable (planar orientation/similar bond distances). ${ }^{30}$ It is reasonable that $\mathrm{K}^{+}$could occupy both fully watercoordinated and partial nitrogen-coordinated positions. 

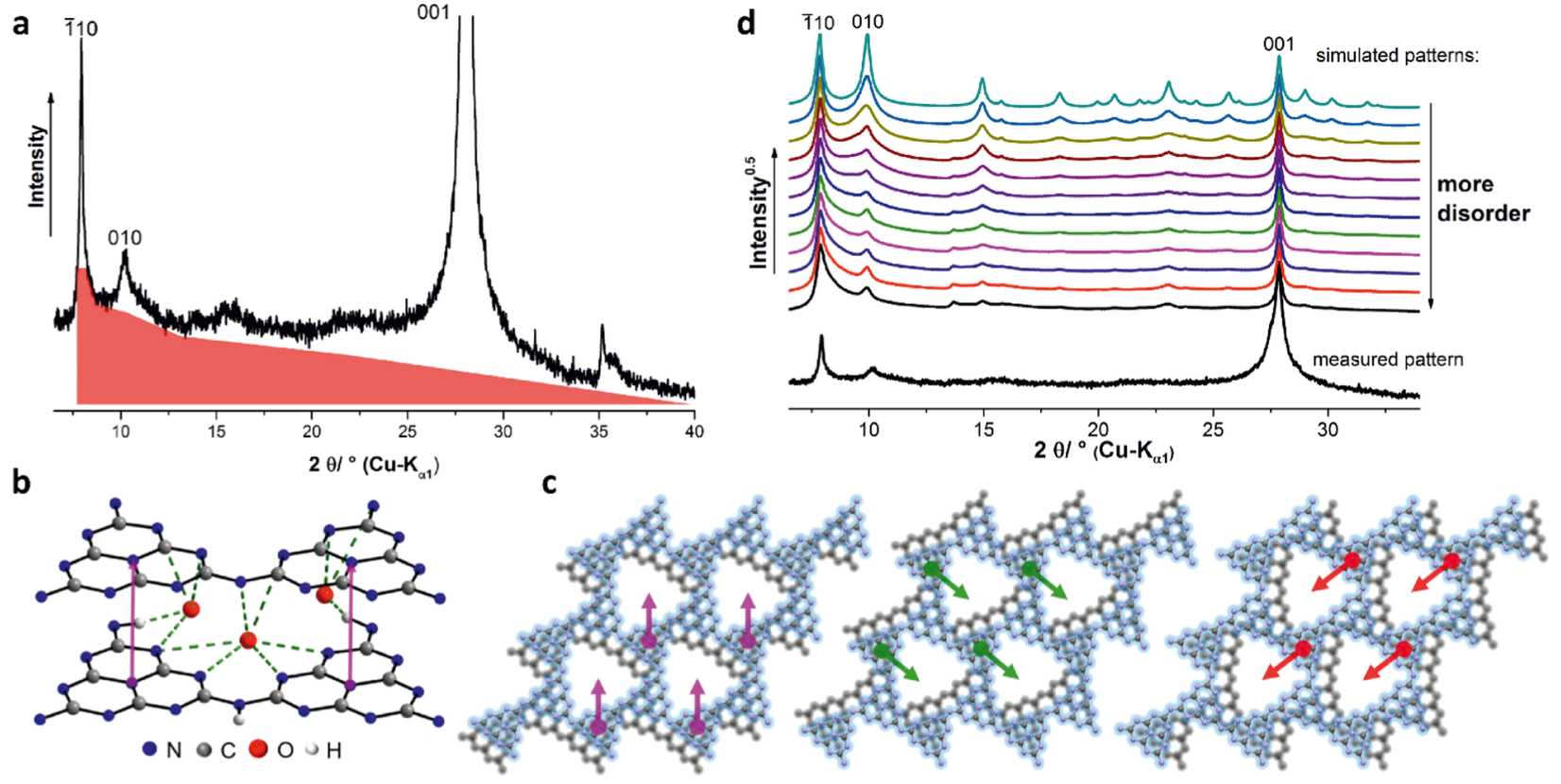

Figure 4. (a) XRPD of H-PHI showing the triangular peak shape. (b) Model with suggested influence of pore material (in this case, water, Figure S40), leading to indirect interactions between the layers as well as different stacking variants with respective colored stacking vectors. (c) Possible equivalent stacking vectors for sheets of PHI; (d) simulation for faulting scenario IV (see chapter 12 of the SI and Figures S47-S49).

Both TEM and XRPD data are consistent with the comparably high crystallinity of the carbon nitride backbone. The overall high in-plane order likely results from the use of molecular precursors that have a higher mobility in the salt melt, which serves as a source for error correction during bond formation. The fact that the reflections in the XRPD pattern of $\mathrm{H}-\mathrm{PHI}$ are particularly broadened will be discussed in the next section.

Stacking Fault Simulations. Information on the 3D structure of the bulk PHI is difficult to extract from electron diffraction patterns due to the local nature of the method and the pronounced 2D morphology of the crystallites. The structural coherence of the PHI materials in the third dimension is governed by the stacking order of the layers and in the case of H-PHI is vastly affected by planar defects occurring as stacking faults. Thus, systematic simulations ${ }^{31}$ of distinct faulting scenarios have been performed and compared qualitatively to the measured XRPD pattern, which yields information on all three dimensions of the bulk material. $\mathrm{H}$ PHI in Figure 4a shows significant peak broadening and triangular, Warren-type line shapes, ${ }^{32}$ which are indicative of planar defects in the crystal structure. We therefore conclude that H-PHI has a higher degree of out-of-plane disorder compared with K-PHI.

In Figure $4 b$, possible interactions of pore water with the poly(heptazine imide) backbone of H-PHI are depicted. These interactions direct the stacking of the layers and induce a layer offset; i.e., a layer is shifted toward the pore of the preceding layer. Due to the intrinsic trigonal symmetry of the heptazine subunit, this type of stacking exhibits three equivalent directions (Figure 4c). The eclipsed case was also considered for the simulations, i.e., layers stacked directly atop each other. Different faulting scenarios were developed using these four stacking relationships and used for qualitative simulations on the diffractional effect of structural disorder (detailed information is given in chapter 12 of the SI). The faulting scenario ("Faulting scenario IV", Figures S47-S49) that was evaluated as the most suitable based on the match between simulated and measured patterns describes completely random transitions between all three pore directed stackings. This leads to a loss in the orientational coherence perpendicular to the layer planes and therefore to vast broadening of most reflections in the XRPD pattern in Figure 4d. Small sections of homogenously stacked layers are, however, still apparent, as indicated by the presence of the remaining 010 reflection.

The combined approach of PDF and XRPD along with stacking fault simulations reveals the overall $3 \mathrm{D}$ structures of H-PHI and K-PHI. It has been shown that the stacking of the pores is governed by rather weak pore-water interactions in the case of H-PHI, resulting in highly defective stacking. Nevertheless, pore water clearly mediates interlayer interaction. When the water molecules are removed by heating $\mathrm{H}$ PHI up to $400{ }^{\circ} \mathrm{C}$ under an inert, dynamic atmosphere in a TG-experiment, an ex situ XRPD analysis of the residue (Figure S27) reveals a significant decrease in the crystallite size perpendicular to the layer planes. The complete removal of pore water also shifts the layer arrangement toward an eclipsed stacking order. The presence of potassium within the pores leads to a more ordered stacking, which is indicated by much sharper reflections in the corresponding XRPD pattern (see Figure 3a). Therefore, the range of the interlayer interaction is much larger in K-PHI than in H-PHI. This can be attributed both to the large ionic radius of the potassium cation and its more isolated positive charge. As potassium is located offcenter in the pores, it interacts with the PHI framework. In addition, a partial hydration sphere is formed around the cation by the pore water molecules (Figure S25), which may indirectly mediate interactions to further distant layers.

Photocatalysis. Knowledge of the structure-property relationships in this class of carbon nitrides is key for the rational design of photocatalysts with improved properties. We therefore investigated the photocatalytic activity of $\mathrm{H}-\mathrm{PHI}$ and K-PHI, obtained from ampoule synthesis, for the hydrogen evolution reaction (HER) with a focus on carving out those 
structural features that affect the activity the most. We find that $\mathrm{H}-\mathrm{PHI}$, like K-PHI, is active toward HER in the presence of a $\mathrm{Pt}$ cocatalyst and methanol as the sacrificial electron donor but shows only moderate activity (H-PHI: $88 \mu \mathrm{mol} / \mathrm{gh}, \mathrm{K}-\mathrm{PHI}$ : $193 \mu \mathrm{mol} / \mathrm{gh}$ ). This can be directly traced back to the large crystallites that tend to form aggregates that disperse only weakly in aqueous solution, thus having less accessible surface area and a reduced number of edge sites available. The latter seems to be vital for the catalytic activity ${ }^{33}$ for cocatalystsupported systems through the presence of cyanamide terminations at the edges of the platelets. These terminations can bind more efficiently to the $\mathrm{Pt}$ cocatalyst and provide a higher degree of ionicity and thus colloidal stability. ${ }^{2}$

To test this hypothesis, we synthesized variants of $\mathrm{H}-\mathrm{PHI}$ and K-PHI with smaller crystallite sizes $(\sim 20 \mathrm{~nm})$ and an increased amount of external cyanamide groups accordingly, by using a mixture of melon and $\mathrm{KSCN}$ as precursors at ambient pressure under an argon flow, followed by treatment in diluted $\mathrm{HCl}$. For the as-obtained, optimized K-PHI, we recorded a 3fold increased activity of $600 \mu \mathrm{mol} / \mathrm{gh}$ compared to that of the highly crystalline sample, whereas we registered a 38-time increase in activity for H-PHI $(3364 \mu \mathrm{mol} / \mathrm{gh}$, Figure $5 \mathrm{~b})$. This

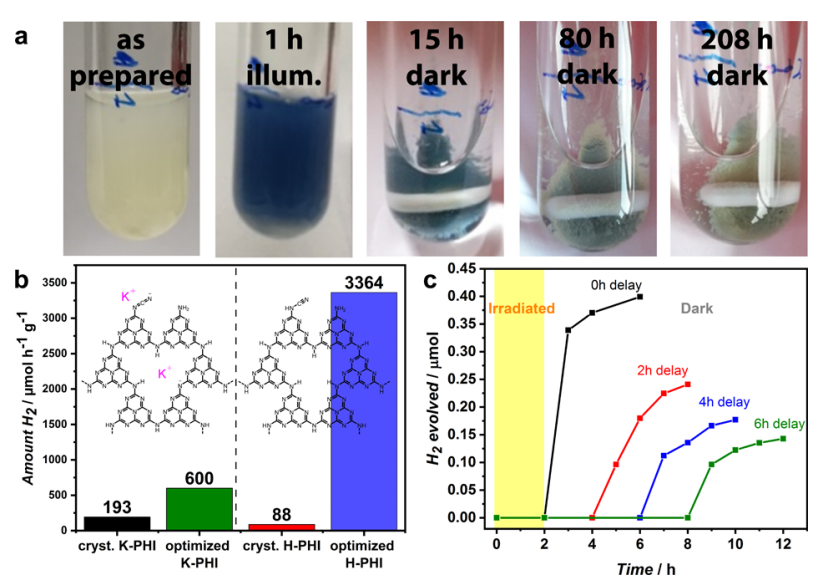

Figure 5. (a) H-PHI before and after $1 \mathrm{~h}$ of illumination and after storing the material in the stable, photoreduced "blue" state up to 208 $\mathrm{h}$ in the dark. A shade toward green is likely due to small amounts of oxygen leaking into the vessel. (b) Comparison of photocatalytic rates for hydrogen evolution for K-PHI and H-PHI, respectively. (c) Dark photocatalysis in phosphate buffer with various delay times for addition of platinum cocatalyst, i.e., 0,2 , 4, or $6 \mathrm{~h}$.

is well beyond the activity of the prototypical carbon nitride melon with an activity of just $25 \mu \mathrm{mol} / \mathrm{gh}$ measured under the same conditions. Note, however, that a quantitative comparison of the intrinsic photocatalytic activities of K-PHI and H$\mathrm{PHI}$ is difficult since the observed hydrogen evolution rates depend on a complex interplay between various parameters, which, in addition, may scale differently with the particle size. These include exfoliation and agglomeration of the crystallites, the nature and amount of defects, or the amount of cocatalyst incorporated in the sample.

For a more detailed structural characterization of these optimized compounds, see Figures S7 and S8. It is apparent that the optimized materials exhibit lower long-range in-plane order, as indicated by broad, diffuse reflections. In particular, both $\overline{1} 10$ and the 010 reflections are vastly broadened, which is attributed to a loss of coherence within the layer plane, i.e., smaller platelets (Figure S5). By using the Scherrer equation from the XRPD data in Figure S8, the crystallite sizes in the lateral dimension have been estimated to be about $20 \mathrm{~nm}$ for the optimized samples and up to $100 \mathrm{~nm}$ (Table S1) for the most crystalline samples. The extension in $c$-direction remains fairly similar for all samples at approx. $20 \mathrm{~nm}$. Small crystallites have a higher amount of accessible cyanamide groups at their surface and are suspended more easily in water, enabling them to participate more efficiently in the photocatalytic reaction.

While the HER activity observed for H-PHI puts it on par with the best carbon nitride photocatalysts, ${ }^{34-39}$ it bears another intriguing property, which has previously been documented for K-PHI; ${ }^{4,6}$ under illumination and in the presence of an electron donor, $\mathrm{H}$-PHI also forms a photoreduced state that is exceptionally long-living, clearly visible by its blue color. Figure 5a depicts the suspension during photoreduction, which is yellow initially and then turns dark blue upon irradiation in the presence of 4-methyl benzylalcohol as the electron donor. Under an argon atmosphere, the photoexcited photocatalyst is reduced by the electron donor and remains in the photoreduced state for over $200 \mathrm{~h}$. The diminishing of the blue color is due to quenching of the anion radical by oxygen, which slowly diffuses into the solution over time (Figure 5a, right). The photoreduced radical state was also monitored by electron magnetic resonance spectroscopy (EPR), which shows a photoinduced paramagnetic signal for both K-PHI and H-PHI with g-factors of $\approx 2.003$ (Figure S10), indicative of a heptazine-centered $\pi$ radical. $^{4,36,40,41}$ To test whether photoreduced $\mathrm{H}-\mathrm{PHI}$ is able to store the trapped electrons and release them upon addition of a hydrogen evolution catalyst after a time delay, $15 \mathrm{mg}$ of the sample was irradiated under a Xenon lamp $\left(100 \mathrm{~mW} / \mathrm{cm}^{2}, \mathrm{AM}\right.$ $1.5 \mathrm{G}$ ) in phosphate buffer with 4-methylbenzyl alcohol as an electron donor for $2 \mathrm{~h}$. Then, a colloidal platinum suspension was added in the dark (Figure 5c) either directly or 2, 4, or $6 \mathrm{~h}$ after illumination. Indeed, hydrogen was evolved for several hours $(27 \mu \mathrm{mol} / \mathrm{g}$, direct addition), which shows the ability of the system to separate the light (photoexcitation) from the dark reaction (catalysis) akin to the biological model of photosynthesis. Even after waiting for 6 h, 37\% of the initial amount of $\mathrm{H}_{2}$ could still be registered.

\section{CONCLUSIONS}

In conclusion, we have synthesized a $2 \mathrm{D}$ poly(heptazine imide) (PHI) polymer, which is comparable with PTI . $\mathrm{LiCl}^{11,12}$ in terms of crystallinity but by far exceeds its photocatalytic performance. ${ }^{13,42}$ Characterization of K-PHI and H-PHI provides insights into the structure-propertyactivity relationship of this new class of high-performance photocatalysts. By combining a suite of analytical techniques, including TEM, NMR spectroscopy, XRPD, PDF analysis, and quantum-chemical calculations, we were able to confirm the inplane PHI-based 2D structure and derive a detailed picture of the out-of-plane structure. Our analysis not only suggests that ionothermal synthesis conditions likely support error correction, thus leading to extended crystallites of over $100 \mathrm{~nm}$ in lateral dimension, but also that metal ions can act as out-ofplane structure-directing agents, leading to a pronounced longrange order in the $c$-direction. Furthermore, water inside the pores has been found to have a major influence on the 3D stacking structure of PHI networks. Our work thus adds another structurally characterized member to the large yet illdefined class of carbon nitrides, which is notoriously difficult to characterize. As such, this study provides synthetic and 
analytical tools that are crucial for the rational design of better photocatalysts and helps to carve out the structural features that are essential for observing an unusually long-lived charge separated state, enabling time-delayed photocatalysis in the dark.

\section{EXPERIMENTAL SECTION}

Crystalline Ampoule Synthesis of Potassium Poly(Heptazine Imide), K-PHI. Dicyandiamide (97 mg, $1.14 \mathrm{mmol}$, Sigma-Aldrich >98\%), KSCN (18 mg, $0.19 \mathrm{mmol}$, Gruessing 99\%), and potassium melonate penta-hydrate $(79 \mathrm{mg}, 0.16 \mathrm{mmol})$ were ground in a mortar and transferred into a Duran glas ampoule, which was subsequently purged four times with argon and then dried under high vacuum. The ampoule was then sealed and placed into a tube furnace $\left(6{ }^{\circ} \mathrm{C} / \mathrm{min}, 500{ }^{\circ} \mathrm{C}, 6 \mathrm{~h}, 1{ }^{\circ} \mathrm{C} / \mathrm{min}\right.$, rt $)$. The bright yellow solid was washed four times with deionized (DI) water and then dried at $60{ }^{\circ} \mathrm{C}$.

Crystalline Ampoule Synthesis of Protonated Poly(Heptazine Imide), H-PHI. Finely ground cryst. K-PHI (100 mg) was treated with $100 \mathrm{~mL}$ of $2 \mathrm{M} \mathrm{HCl}$ and stirred for $15 \mathrm{~min}$. The suspension was filtered and the residue washed four times with DI water to yield $\mathrm{H}$-PHI.

Further details on the synthesis of optimized samples for photocatalysis, melon, and potassium melonate can be found in Supporting Information, chapter 1.

\section{ASSOCIATED CONTENT}

\section{S Supporting Information}

The Supporting Information is available free of charge on the ACS Publications website at DOI: 10.1021/acs.chemmater.9b02199.

Further experimental details, instrumental details, additional characterization (TEM, IR, Raman, XRPD (XLSX), TG-XRPD (XLSX), crystallite size estimations, EPR, ssNMR, TGA, PDF analysis, quantum-chemical calculations and methods (XLSX), recursive-type stacking simulations, EA and water sorption) (PDF)

Structural model for K-PHI from Rietveld refinement (CIF)

Structural model for H-PHI from PDF refinement (CIF)

\section{AUTHOR INFORMATION}

\section{Corresponding Author}

*E-mail: b.lotsch@fkf.mpg.de.

\section{ORCID}

Hendrik Schlomberg: 0000-0001-8712-0942

Julia Kröger: 0000-0002-6163-7625

Gökcen Savasci: 0000-0002-6183-7715

Maxwell W. Terban: 0000-0002-7094-1266

Sebastian Bette: 0000-0003-3575-0517

Filip Podjaski: 0000-0002-5428-4526

Jürgen Senker: 0000-0002-7278-7952

Christian Ochsenfeld: 0000-0002-4189-6558

Bettina V. Lotsch: 0000-0002-3094-303X

\section{Author Contributions}

${ }^{\perp}$ H.S. and J.K. contributed equally to this work.

\section{Funding}

Financial support is gratefully acknowledged from the Max Planck Society, the ERC Starting Grant (project COFLeaf, grant number 639233), the Center for NanoScience (CeNS), the German Research Foundation (SFB 840), and the BASF.

\section{Notes}

The authors declare no competing financial interest.

\section{ACKNOWLEDGMENTS}

Financial support by the ERC Starting Grant (project COF Leaf, grant number 639233), the Max Planck Society, the DFG cluster of excellence "e-conversion", and the Center for NanoScience (CeNS) is gratefully acknowledged. C.O. acknowledges financial support as a Max Planck Fellow at the Max Planck Institute for Solid State Research, Stuttgart. M.W.T. acknowledges support from BASF. This research used beamline 28-ID-2 of the National Synchrotron Light Source II, a U.S. Department of Energy (DOE) Office of the Science User Facility operated for the DOE Office of Science by Brookhaven National Laboratory under Contract no. DESC0012704. The authors thank Agnieszka Poulain for assistance with experiments performed on beamline ID-31 at the European Synchrotron Radiation Facility (ESRF), Grenoble, France. R.S. and J.S. gratefully acknowledge financial support from the German Research Foundation (SFB 840). We thank Torsten Gutmann for support with the DNP NMR measurements and Prof. Gerd Buntkowsky (TU Darmstadt) for generous allocation of measurement time at his Bruker Avance III 400 DNP spectrometer. The authors further thank Stefan Trenker and Alberto von Mankowski for water sorption measurements as well as Armin Schulz for Raman spectra.

\section{REFERENCES}

(1) Savateev, A.; Dontsova, D.; Kurpil, B.; Antonietti, M. Highly crystalline poly(heptazine imides) by mechanochemical synthesis for photooxidation of various organic substrates using an intriguing electron acceptor - Elemental sulfur. J. Catal. 2017, 350, 203-211.

(2) Lau, V. W.-h.; Moudrakovski, I.; Botari, T.; Weinberger, S.; Mesch, M. B.; Duppel, V.; Senker, J.; Blum, V.; Lotsch, B. V. Rational design of carbon nitride photocatalysts by identification of cyanamide defects as catalytically relevant sites. Nat. Commun. 2016, 7, No. 12165 .

(3) Ou, H.; Lin, L.; Zheng, Y.; Yang, P.; Fang, Y.; Wang, X. Tri-striazine-Based Crystalline Carbon Nitride Nanosheets for an Improved Hydrogen Evolution. Adv. Mater. 2017, 29, No. 1700008.

(4) Lau, V. W.-h.; Klose, D.; Kasap, H.; Podjaski, F.; Pignié, M.-C.; Reisner, E.; Jeschke, G.; Lotsch, B. V. Dark Photocatalysis: Storage of Solar Energy in Carbon Nitride for Time-Delayed Hydrogen Generation. Angew. Chem., Int. Ed. 2017, 56, 510-514.

(5) Podjaski, F.; Kröger, J.; Lotsch, B. V. Toward an Aqueous Solar Battery: Direct Electrochemical Storage of Solar Energy in Carbon Nitrides. Adv. Mater. 2018, 30, No. 1705477.

(6) Zeng, Z.; Quan, X.; Yu, H.; Chen, S.; Zhang, Y.; Zhao, H.; Zhang, S. Carbon nitride with electron storage property: Enhanced exciton dissociation for high-efficient photocatalysis. Appl. Catal., B 2018, 236, 99-106.

(7) Savateev, A.; Kurpil, B.; Mishchenko, A.; Zhang, G.; Antonietti, M. A“waiting" carbon nitride radical anion: A charge storage material and key intermediate in direct $\mathrm{C}-\mathrm{H}$ thiolation of methylarenes using elemental sulfur as the "S"-source. Chem. Sci. 2018, 9, 3584-3591.

(8) Chen, Z.; Savateev, A.; Pronkin, S.; Papaefthimiou, V.; Wolff, C.; Willinger, M. G.; Willinger, E.; Neher, D.; Antonietti, M.; Dontsova, D. "The Easier the Better" Preparation of Efficient PhotocatalystsMetastable Poly(heptazine imide) Salts. Adv. Mater. 2017, 8, No. 1700555

(9) Savateev, A.; Pronkin, S.; Willinger, M. G.; Antonietti, M.; Dontsova, D. Towards Organic Zeolites and Inclusion Catalysts: Heptazine Imide Salts Can Exchange Metal Cations in the Solid State. Chem. Asian J. 2017, 12, 1517.

(10) Lin, L.; Ren, W.; Wang, C.; Asiri, A. M.; Zhang, J.; Wang, X. Crystalline carbon nitride semiconductors prepared at different 
temperatures for photocatalytic hydrogen production. Appl. Catal., B 2018, 231, 234-241.

(11) Wirnhier, E.; Döblinger, M.; Gunzelmann, D.; Senker, J.; Lotsch, B. V.; Schnick, W. Poly(triazine imide) with Intercalation of Lithium and Chloride Ions [(C3N3)2(NHxLil-x)3.LiCl]: A Crystalline 2D Carbon Nitride Network. Chem. - Eur. J. 2011, 17, $3213-3221$.

(12) Mesch, M. B.; Bärwinkel, K.; Krysiak, Y.; Martineau, C.; Taulelle, F.; Neder, R. B.; Kolb, U.; Senker, J. Solving the Hydrogen and Lithium Substructure of Poly(triazine imide)/LiCl Using NMR Crystallography. Chem. - Eur. J. 2016, 22, 16878-16890.

(13) Ham, Y.; Maeda, K.; Cha, D.; Takanabe, K.; Domen, K. Synthesis and Photocatalytic Activity of Poly(triazine imide). Chem. Asian J. 2013, 8, 218-224.

(14) Iwama, M.; Suzuki, Y.; Plévert, J.; Itabashi, K.; Ogura, M.; Okubo, T. Location of Alkali Ions and their Relevance to Crystallization of Low Silica X Zeolite. Cryst. Growth Des. 2010, 10, 3471-3479.

(15) Döblinger, M.; Lotsch, B. V.; Wack, J.; Thun, J.; Senker, J.; Schnick, W. Structure elucidation of polyheptazine imide by electron diffraction-a templated 2D carbon nitride network. Chem. Commun. 2009, 1541 .

(16) Schaefer, J.; McKay, R. A.; Stejskal, E. O. Double-crosspolarization NMR of solids. J. Magn. Reson. 1979, 34, 443-447.

(17) Levitt, M. H. Symmetry-based pulse sequences in magic-angle spinning solid-state NMR. In Encyclopedia of Nuclear Magnetic Resonance; Grant, D. M.; Harris, R. K., Eds.; Wiley: Chichester, U.K., 2007; Vol. 9, pp 165-196.

(18) Adamo, C.; Barone, V. Toward reliable density functional methods without adjustable parameters: The PBE0 model. J. Chem. Phys. 1999, 110, 6158-6170.

(19) Ernzerhof, M.; Scuseria, G. E. Assessment of the PerdewBurke-Ernzerhof exchange-correlation functional. J. Chem. Phys. 1999, 110, 5029-5036.

(20) Grimme, S.; Antony, J.; Ehrlich, S.; Krieg, H. A consistent and accurate $\mathrm{ab}$ initio parametrization of density functional dispersion correction (DFT-D) for the 94 elements H-Pu. J. Chem. Phys. 2010, 132, No. 154104.

(21) Schäfer, A.; Huber, C.; Ahlrichs, R. Fully optimized contracted Gaussian basis sets of triple zeta valence quality for atoms Li to Kr. J. Chem. Phys. 1994, 100, 5829-5835.

(22) Wilson, P. J.; Bradley, T. J.; Tozer, D. J. Hybrid exchangecorrelation functional determined from thermochemical data and $a b$ initio potentials. J. Chem. Phys. 2001, 115, 9233-9242.

(23) Jensen, F. Segmented contracted basis sets optimized for nuclear magnetic shielding. J. Chem. Theory Comput. 2015, 11, 132138.

(24) Jürgens, B.; Irran, E.; Senker, J.; Kroll, P.; Müller, H.; Schnick, W. Melem (2,5,8-Triamino-tri- $s$-triazine), an Important Intermediate during Condensation of Melamine Rings to Graphitic Carbon Nitride: Synthesis, Structure Determination by X-ray Powder Diffractometry, Solid-State NMR, and Theoretical Studies. J. Am. Chem. Soc. 2003, 125, 10288-10300.

(25) Horvath-Bordon, E.; Kroke, E.; Svoboda, I.; Fuess, H.; Riedel, R. Potassium melonate, $\mathrm{K} 3[\mathrm{C} 6 \mathrm{~N} 7(\mathrm{NCN}) 3] \cdot 5 \mathrm{H} 2 \mathrm{O}$, and its potential use for the synthesis of graphite-like C3N4 materials. New J. Chem. 2005, 29, 693.

(26) Sattler, A.; Schnick, W. Zur Frage der Tautomerie von Cyamelursäure im Kristall. Z. Anorg. Allg. Chem. 2006, 632, 15181523.

(27) Makowski, S. J.; Gunzelmann, D.; Senker, J.; Schnick, W. Protonated Melonate $\mathrm{Ca}\left[\mathrm{HC}_{6} \mathrm{~N}_{7}(\mathrm{NCN})_{3}\right] \cdot 7 \mathrm{H}_{2} \mathrm{O}$ - Synthesis, Crystal Structure, and Thermal Properties. Z. Anorg. Allg. Chem. 2009, 635, 2434-2439.

(28) Pawley, G. S. Unit-cell refinement from powder diffraction scans. J. Appl. Crystallogr. 1981, 14, 357-361.

(29) Rietveld, H. M. A profile refinement method for nuclear and magnetic structures. J. Appl. Crystallogr. 1969, 2, 65-71.
(30) Amunugama, R.; Rodgers, M. T. Absolute alkali metal ion binding affinities of several azines determined by threshold collisioninduced dissociation and ab initio theory. Int. J. Mass Spectrom. 2000, 195-196, 439-457.

(31) Coelho, A. A.; Evans, J. S. O.; Lewis, J. W. Averaging the intensity of many-layered structures for accurate stacking-fault analysis using Rietveld refinement. J. Appl. Crystallogr. 2016, 49, 1740-1749.

(32) Warren, B. E. X-Ray Diffraction in Random Layer Lattices. Phys. Rev. 1941, 59, 693-698.

(33) Haiber, D. M.; Crozier, P. A. Nanoscale Probing of Local Hydrogen Heterogeneity in Disordered Carbon Nitrides with Vibrational Electron Energy-Loss Spectroscopy. ACS Nano 2018, $12,5463-5472$.

(34) Liu, G.; Wang, T.; Zhang, H.; Meng, X.; Hao, D.; Chang, K.; Li, P.; Kako, T.; Ye, J. Nature-Inspired Environmental "Phosphorylation" Boosts Photocatalytic H2 Production over Carbon Nitride Nanosheets under Visible-Light Irradiation. Angew. Chem., Int. Ed. 2015, 54, 13561-13565.

(35) Martin, D. J.; Qiu, K.; Shevlin, S. A.; Handoko, A. D.; Chen, X.; Guo, Z.; Tang, J. Highly Efficient Photocatalytic H2 Evolution from Water using Visible Light and Structure-Controlled Graphitic Carbon Nitride. Angew. Chem., Int. Ed. 2014, 53, 9240-9245.

(36) Sun, J.; Zhang, J.; Zhang, M.; Antonietti, M.; Fu, X.; Wang, X. Bioinspired hollow semiconductor nanospheres as photosynthetic nanoparticles. Nat. Commun. 2012, 3, No. 711.

(37) Kurpil, B.; Savateev, A.; Papaefthimiou, V.; Zafeiratos, S.; Heil, T.; Özenler, S.; Dontsova, D.; Antonietti, M. Hexaazatriphenylene doped carbon nitrides-Biomimetic photocatalyst with superior oxidation power. Appl. Catal., B 2017, 217, 622-628.

(38) Lau, V. W.-h.; Yu, V. W.-z.; Ehrat, F.; Botari, T.; Moudrakovski, I.; Simon, T.; Duppel, V.; Medina, E.; Stolarczyk, J.; Feldmann, J.; et al. Urea-Modified Carbon Nitrides: Enhancing Photocatalytic Hydrogen Evolution by Rational Defect Engineering. Adv. Energy Mater. 2017, 7, No. 1602251.

(39) Cui, L.; Song, J.; McGuire, A. F.; Kang, S.; Fang, X.; Wang, J.; Yin, C.; Li, X.; Wang, Y.; Cui, B. Constructing Highly Uniform Onion-Ring-like Graphitic Carbon Nitride for Efficient Visible-LightDriven Photocatalytic Hydrogen Evolution. ACS Nano 2018, 12, $5551-5558$.

(40) Dvoranová, D.; Mazúr, M.; Papailias, I.; Giannakopoulou, T.; Trapalis, C.; Brezová, V. EPR Investigations of G-C3N4/TiO2 Nanocomposites. Catalysts 2018, 8, No. 47.

(41) Rodríguez, N. A.; Savateev, A.; Grela, M. A.; Dontsova, D. Facile Synthesis of Potassium Poly(heptazine imide) (PHIK)/TiBased Metal-Organic Framework (MIL-125-NH2) Composites for Photocatalytic Applications. ACS Appl. Mater. Interfaces 2017, 9, 22941-22949.

(42) Schwinghammer, K.; Tuffy, B.; Mesch, M. B.; Wirnhier, E.; Martineau, C.; Taulelle, F.; Schnick, W.; Senker, J.; Lotsch, B. V. Triazine-based Carbon Nitrides for Visible-Light-Driven Hydrogen Evolution. Angew. Chem., Int. Ed. 2013, 52, 2435-2439. 